\title{
COVID-19 pneumonia with night sweat as the first symptom
}

\author{
Bangyan Zhang, ${ }^{1, \#}$ Xianwei Ye ${ }^{1, \#, *}, \mathrm{Li} \mathrm{Ma}^{1}, \mathrm{Li}_{\mathrm{Zhao}}{ }^{1}$, Yanju Xu ${ }^{1}$, Shanshan Rao ${ }^{1}$, Xiangyan Zhang ${ }^{1}$, Yiling Tang ${ }^{1,2}$ \\ ${ }^{1}$ Department of Respiratory and Critical Care Medicine, Guizhou Provincial People's Hospital, State Key Laboratory of Diagnosis and \\ Treatment of Lung Immune Diseases, Guiyang, Guizhou 550002, China \\ ${ }^{2}$ ZunYi Medical University, Zunyi, Guizhou 563003, China \\ \# These authors contributed equally to this work. \\ * Correspondence: Xianwei Ye \\ E-mail: yxw1205@163.com \\ Received: March 21, 2020 \\ Published: June 17, 2020
}

\begin{abstract}
A cluster of patients with coronavirus disease 2019 (COVID-19) pneumonia caused by infection with severe acute respiratory syndrome coronavirus 2 (SARS-CoV-2) was successively reported in Wuhan, China. The major presentations of patients are fever, fatigue, and dry cough. No cases have been reported with night sweats as the first symptom. This article presents a case that was diagnosed with COVID-19 pneumonia with night sweats as the first symptom. Chest CT showed patches of ground-glass opacity, and qRT-PCR results of SARS-CoV-2 were positive. After treatment, the patient did not present with night sweats again, the chest CT showed that the ground-glass opacity was completely absorbed, and the SARS-CoV-2 nucleic acid test results were negative. This case suggests that night sweats might be the first and major symptom of COVID-19 pneumonia.
\end{abstract}

Keywords: COVID-19 pneumonia, SARS-CoV-2, night sweats

Abbreviations: COVID-19, coronavirus disease 2019; SARS-CoV-2, severe acute respiratory syndrome coronavirus 2; $C D C$, Centers for Disease Control; $C T$, Computed tomography

To cite this article: Bangyan Zhang, et al. COVID-19 pneumonia with night sweat as the first symptom. Inflamm Cell Signal 2020; 7: e463. doi: 10.14800/ics.463.

\section{Introduction}

Since December 2019, a number of coronavirus disease 2019 (COVID-19) pneumonia patients have been diagnosed in Wuhan, Hubei Province, China. With the spread of the epidemic ${ }^{[1]}$, such cases have been found in other regions in China and abroad. Of the more than 3000,000 cases reported, the major presentations of patients are fever, fatigue, and dry cough. Other symptoms include nasal congestion, runny nose, and diarrhea. No cases have been reported with night sweats as the first symptom. This case study reports a patient diagnosed with COVID-19 pneumonia with night sweats as the first symptom. Night sweats ceased after 10 days of treatment (lopinavir and ritonavir tablets and recombinant human interferon alb), and a nucleic acid test results for the virus were sequentially negative twice. Thus, this is the first report of a patient diagnosed with COVID-19 pneumonia with night sweats as the first symptom.

\section{Case Description}

A 19-year-old young male patient diagnosed with COVID19 pneumonia was admitted to the hospital mainly because of night sweats. During his study in Wuhan, Hubei Province, the patient presented with night sweats for more than one month without obvious cause. No observations of fever, cough, expectoration, fatigue, poor appetite, emaciation, dyspnea, or other symptoms were reported ${ }^{[2-5]}$. On January 14,2020 , the patient returned to Guiding County, Guizhou Province, but still had night sweats. Because his father had previously suffered from tuberculosis, it was recommended that the patient undergo further examinations. Therefore, the patient 
http://www.smartscitech.com/index.php/ics

Table 1. Patient laboratory characteristics.

\begin{tabular}{|c|c|c|c|c|c|c|}
\hline & Reference & & & & & \\
\hline Measure & Range & Hospital Day 1 & Hospital Day 10 & Hospital Day 12 & Hospital Day 14 & Hospital Day 17 \\
\hline White-cell count $\left(10^{9} / \mathrm{L} \quad\right)$ & 4-10 & 4.15 & 4.95 & 4.19 & 4.08 & 5.64 \\
\hline Red-cell count $\left(10^{12} / \mathrm{L}\right)$ & $4-5.5$ & 4.67 & 9.23 & 5.01 & 5 & 4.99 \\
\hline Absolute neutrophil count $\left(10^{9} / \mathrm{L}\right)$ & $2-7$ & 2.3 & 6.72 & 2.07 & 2.09 & 3.32 \\
\hline Absolute lymphocyte count $\left(10^{9} / \mathrm{L}\right)$ & $0.80-4.00$ & 1.12 & 1.93 & 1.7 & 1.54 & 1.88 \\
\hline Platelet $\operatorname{count}\left(10^{9} / \mathrm{L}\right)$ & $100-300$ & 159 & 198 & 204 & 211 & 209 \\
\hline $\operatorname{Hemoglobin}(\mathrm{g} / \mathrm{L})$ & $120-160$ & 145 & 157 & 158 & 155 & 152 \\
\hline Sodium(mmol/liter) & $132-150$ & 159 & 143.2 & 141.8 & 142.1 & - \\
\hline Potassium(mmol/liter) & $3.5-5.3$ & 4.16 & 3.95 & 4.33 & 4.95 & - \\
\hline Chloride(mmol/liter) & $96-108$ & 102.5 & 101.2 & 98.7 & 100.5 & - \\
\hline Alanine aminotransferase(U/L) & $5-40$ & 11.5 & 27.2 & 7 & 8 & 11 \\
\hline Aspartate aminotransferase(U/L) & $8-40$ & 18.1 & 18.2 & 23 & 19 & 21 \\
\hline Albumin $(\mathrm{g} / \mathrm{L})$ & $35-55$ & 49.2 & 53.2 & 51.8 & 50.1 & 47.6 \\
\hline Creatinine (umol/L) & $53-123$ & 81.5 & 82.3 & 95.8 & 87.8 & 68.1 \\
\hline Total bilirubin (umol/L ) & $5.1-20$ & 14.5 & 18.2 & 13.4 & 18.9 & 18.6 \\
\hline $\operatorname{TnI}(\mathrm{ng} / \mathrm{ml})$ & $0-0.3$ & 0.83 & - & 0.05 & - & 0.008 \\
\hline Aspartate aminotransferase (U/L) & $0-190$ & - & - & 112 & 117 & - \\
\hline prothrombin time ( $\mathrm{sec})$ & $11-16$ & - & 13.7 & 13.7 & 12.4 & 13.4 \\
\hline $\mathrm{D}-\operatorname{dimer}(\mathrm{ug} / \mathrm{ml})$ & $0-1$ & - & $0.25^{\#}$ & - & $0.26^{\#}$ & $0.23^{\#}$ \\
\hline erythrocyte sedimentation rate $(\mathrm{mm} / \mathrm{h})$ & $0-15$ & 6 & 5 & 3 & $28^{\#}$ & $21^{\#}$ \\
\hline C-reactive protein $(\mathrm{mg} / \mathrm{L})$ & $0-8$ & 2.1 & 5.3 & 2.14 & $10.81^{\#}$ & $9.46^{\#}$ \\
\hline Procalcitonin(ng/ml) & $0-0.5$ & 0.24 & 0.03 & - & - & 0.027 \\
\hline $\mathrm{PO} 2(\mathrm{mmHg})$ & $83-100$ & - & - & $101^{\#}$ & 96.8 & 38.1 \\
\hline $\mathrm{PCO} 2(\mathrm{mmHg})$ & $35-45$ & - & - & 36.3 & 39.4 & 83.2 \\
\hline
\end{tabular}

\#: The value in the patient was slightly increased.

went to the People's Hospital of Guiding County on January 18,2020 . The medical staff were informed that the patient had returned from a COVID-19 pneumonia epidemic area. Chest CT showed patches of ground-glass opacity in the lateral basal segment of the lower lobe of the right lung (Fig. A), and an oropharyngeal swab was sent to the Centers for Disease Control (CDC) for severe acute respiratory syndrome coronavirus 2 (SARS-CoV-2) testing. On the same day, the patient presented with fever (body temperature $=38^{\circ} \mathrm{C}$ ). After physical cooling and rehydration treatments, his body temperature returned to normal. On January 19, 2020, the patient had intermittent night sweats. On January 20, 2020, the oropharyngeal swab test result for SARS-CoV-2 was negative. On the next day, another oropharyngeal swab was taken and submitted for examination. The result was positive for the virus. On January 22, 2020, it was submitted again to the CDC and reconfirmed to be positive. Physical examination indicated no redness or swelling in the pharynx. Bilateral lung percussion and auscultation indicated no obvious abnormalities. Auxiliary examination showed the complete blood count, C-reactive protein, procalcitonin, erythrocyte sedimentation rate, liver and kidney function, D-dimer, myocardial enzymology, and electrolyte levels were normal (Table 1). Therefore, the patient was diagnosed with COVID19 pneumonia. On January 23, 2020, the patient was treated with lopinavir and ritonavir tablets (2 tablets oral bid) and recombinant human interferon alb (50 $\mu \mathrm{g}$ atomization bid), and the night sweats disappeared after 2 days of treatment. For close isolation treatment, the patient was transferred to Guizhou Jiangjunshan Hospital (formerly Guiyang Public Health Treatment Center) on January 28, 2020. Treatment plans remained unchanged during the whole course. The nucleic acid test results for SARS-CoV-2 on February 1, 2020, were negative and were negative again the next day. Chest CT was normal on February 3, 2020. The patient was cured and discharged on February 5, 2020.

\section{Discussion}

According to a recent study ${ }^{[3]}$ published in the Lancet, the most common symptoms at the onset of COVID-19 pneumonia are fever (83\%), cough (82\%), and dyspnea (31\%). Less common symptoms are muscle pain, headache, dizziness, chest pain, and diarrhea, accounting for less than $10 \%$ of 

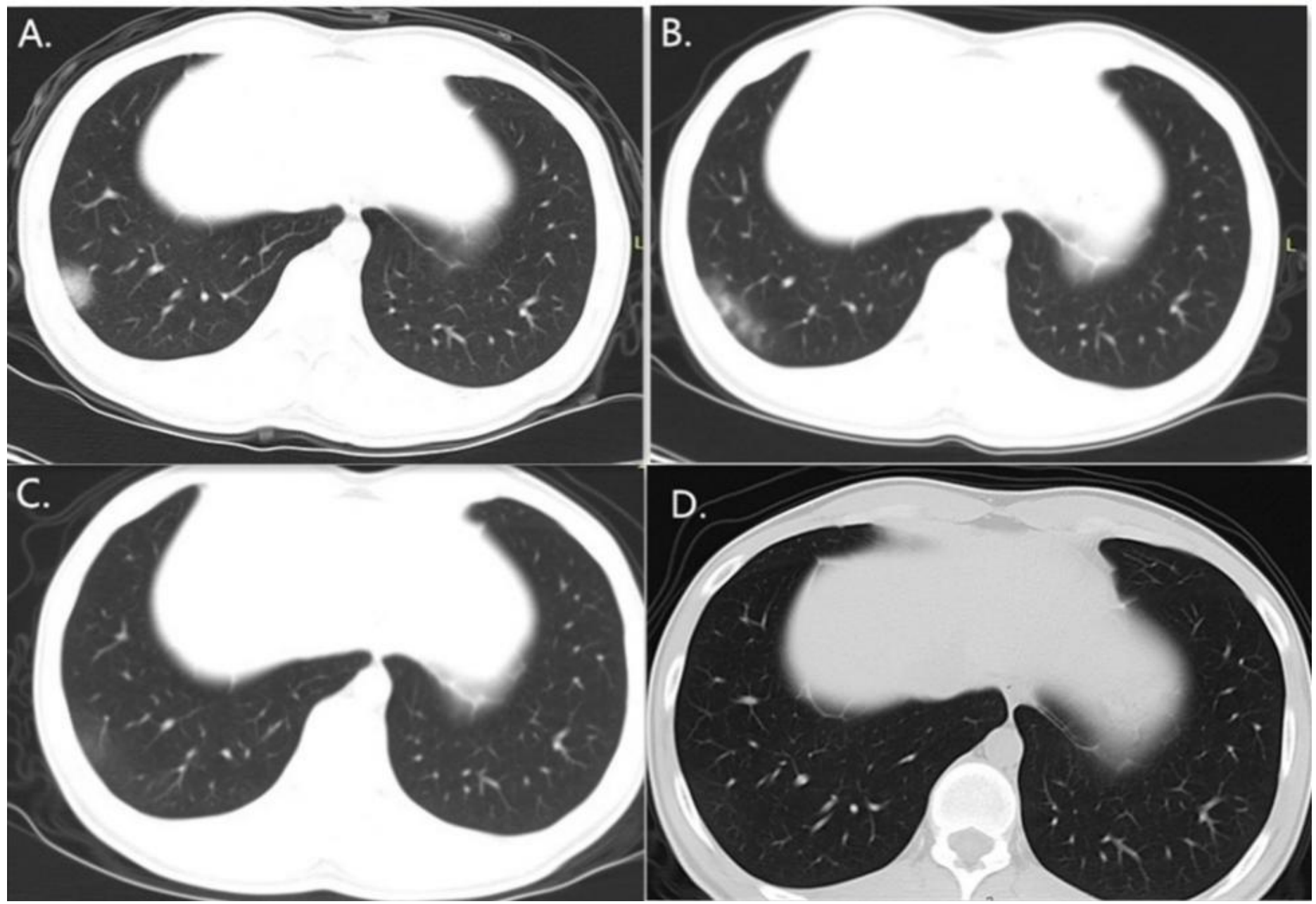

Figure 1. CT images of the chest in the lung window. (A) Chest CT on January 18, 2020, showed patches of ground-glass opacity in the lateral basal segment of the lower lobe of the right lung. (B) Chest CT on January 23, 2020, showed patches of ground-glass opacity in the lateral basal segment of the lower lobe of the right lung was decreasing. (C) Chest CT on January 26, 2020, showed patches of ground-glass opacity that decreased after 3 days of treatment. (D) Chest CT on February 3, 2020, showed that the ground-glass opacity was completely absorbed.

patients. In addition, some studies ${ }^{[4,5]}$ reported that fever, cough, and fatigue were the major clinical symptoms. Currently, there have been no reports that night sweats are the first and major symptom related to COVID-19 pneumonia. Night sweating refers to abnormal sweating after falling asleep and is characterized by sweating cessation after waking up. The etiology mainly includes infection, tumor, endocrine dyscrasia, and medication, of which tuberculosis is the main cause of night sweats in young patients. This patient was a young man whose father suffered from tuberculosis; therefore, he had a history of contact with tuberculosis. Accordingly, tuberculosis should be considered first. The erythrocyte sedimentation rate, purified protein derivative and interferon$\gamma$ release assays were all negative; thus, tuberculosis was excluded. In addition, the patient's history, physical signs, and other auxiliary examinations showed that the night sweats were less likely to be caused by other known diseases.
The first and major symptom of the patient was mild night sweating, which lasted for more than one month. Complete blood count showed that leukocytes and lymphocytes were normal, and that the D-dimer and related infection indexes were normal. According to the characteristics of patients reported so far, the presentation of this patient does not conform to the clinical manifestations of COVID-19 pneumonia. However, results from qRT-PCR analyses of oropharyngeal swabs from the patient for SARS-CoV-2 were positive. After treatment, the patient did not present with night sweats again, the chest CT showed that the ground-glass opacity was completely absorbed (Fig. 1D), and the SARSCoV-2 nucleic acid test result was negative. This case suggests that night sweats might be the first and major symptom of COVID-19 pneumonia. Clinicians should be alerted to pneumonia cases with night sweats and cases from epidemic areas to avoid misdiagnosis and missed diagnosis and to avoid the case developing into a potential infectious source. 
http://www.smartscitech.com/index.php/ics

\section{Ethics Approval}

The study was approved by the Ethics Committee of the Guizhou Provincial Hospital.

\section{Patient Consent for Publication}

A written informed consent was obtained from the patient for publication of the information about him that appears within this Case Report.

\section{Acknowledgements}

We thank American Journal Experts (AJE) for providing the Standard English Editing service.

\section{Funding}

None.

\section{Conflicting Interests}

The authors declare that they have no conflict of interests.

\section{References}

1. Jasper Fuk-Woo Chan, Shuofeng Yuan, Kin-Hang Kok, et al. A familial cluster of pneumonia associated with the 2019 novel coronavirus indicating person-to-person transmission: a study of a family cluster. The Lancet 2020; 395: 514-523.

2. Chaolin Huang, Yeming Wang, Xingwang Li, et al. Clinical features of patients infected with 2019 novel coronavirus in Wuhan, China. The Lancet 2020; 395: 497-506.

3. Nanshan Chen, Min Zhou, Xuan Dong, et al. Epidemiological and clinical characteristics of 99 cases of 2019 novel coronavirus pneumonia in Wuhan, China: a descriptive study. The Lancet 2020; 395: 507-513.

4. Dawei Wang, Bo Hu, Chang Hu, et al. Clinical Characteristics of 138 Hospitalized Patients With 2019 Novel Coronavirus-Infected Pneumonia in Wuhan, China. JAMA [doi:10.1001/jama.2020.1585].

5. Weijie,Guan, Zhengyi Ni, Yu Hu, et al. Clinical characteristics of 2019 novel coronavirus infection in China. MedRxiv 20020974. [doi: 10.1101/2020.02.06.20020974]. 